\title{
Subtyping Lung Cancer Using DNA Methylation in Liquid Biopsies
}

\author{
Sandra P. Nunes ${ }^{1,+}+\mathbb{C}$, Francisca Diniz ${ }^{1,+}+\mathbb{C}$, Catarina Moreira-Barbosa ${ }^{1}$, Vera Constâncio ${ }^{1}(\mathbb{D}$, \\ Ana Victor Silva ${ }^{2}$, Júlio Oliveira ${ }^{2}$, Marta Soares ${ }^{2}$, Sofia Paulino ${ }^{1,3}$, Ana Luísa Cunha 1,3, \\ Jéssica Rodrigues ${ }^{4}$, Luís Antunes $4\left(\mathbb{D}\right.$, Rui Henrique ${ }^{1,3,5, * \mathbb{D}}$ and Carmen Jerónimo ${ }^{1,5, *(\mathbb{D})}$ \\ 1 Cancer Biology \& Epigenetics Group-Research Center, Portuguese Oncology Institute of Porto (CI-IPOP), \\ 4200-072 Porto, Portugal; sandra22nunes@gmail.com (S.P.N.); franciscadiniz93@gmail.com (F.D.); \\ catarina.moreira.barbosa@gmail.com (C.M.-B.); veraconstancio24@gmail.com (V.C.); \\ sofia.paulino@ipoporto.min-saude.pt (S.P.); analuisa.cunha@ipoporto.min-saude.pt (A.L.C.) \\ 2 Lung Cancer Clinic and Department of Medical Oncology, Portuguese Oncology Institute of Porto, \\ 4200-072 Porto, Portugal; ana.v.silva@ipoporto.min-saude.pt (A.V.S.); \\ julio.oliveira@ipoporto.min-saude.pt (J.O.); martasoares@ipoporto.min-saude.pt (M.S.) \\ 3 Department of Pathology, Portuguese Oncology Institute of Porto, 4200-072 Porto, Portugal \\ 4 Department of Epidemiology, Portuguese Oncology Institute of Porto, 4200-072 Porto, Portugal; \\ jessica.rocha.rodrigues@ipoporto.min-saude.pt (J.R.); luis.antunes@ipoporto.min-saude.pt (L.A.) \\ 5 Department of Pathology and Molecular Immunology, Institute of Biomedical Sciences Abel Salazar, \\ University of Porto (ICBAS-UP), 4050-313 Porto, Portugal \\ * Correspondence: rmhenrique@icbas.up.pt (R.H.); carmenjeronimo@ipoporto.min-saude.pt (C.J.); \\ Tel.: +315-225-084-000 (R.H. \& C.J.); Fax: +351-225-084-199 (R.H. \& C.J.) \\ + These authors contributed equally to this work.
}

Received: 7 August 2019; Accepted: 17 September 2019; Published: 19 September 2019

\begin{abstract}
Background: Lung cancer (LCa) is the most frequently diagnosed and lethal cancer worldwide. Histopathological subtyping, which has important therapeutic and prognostic implications, requires material collection through invasive procedures, which might be insufficient to enable definitive diagnosis. Aberrant DNA methylation is an early event in carcinogenesis, detectable in circulating cell-free DNA (ccfDNA). Herein, we aimed to assess methylation of selected genes in ccfDNA from LCa patients and determine its accuracy for tumor subtyping. Methods: Methylation levels of APC, $H O X A 9, R A R \beta 2$, and RASSF1A were assessed in three independent study groups (study group \#1: 152 tissue samples; study group \#2: 129 plasma samples; study group \#3: 28 benign lesions of lung) using quantitative methylation-specific PCR. Associations between gene promoter methylation levels and LCa subtypes were evaluated using non-parametric tests. Receiver operating characteristic (ROC) curve analysis was performed. Results: In study group \#2, HOXA9 and RASSF1A displayed higher methylation levels in small-cell lung cancer (SCLC) than in non-small-cell lung cancer (NSCLC). HOXA9 displayed high sensitivity (63.8\%), whereas RASSF1A disclosed high specificity $(96.2 \%)$ for SCLC detection in ccfDNA. Furthermore, HOXA9 methylation levels showed to be higher in squamous cell carcinoma in comparison with adenocarcinoma in study group \#1. Conclusions: Methylation level assessments in ccfDNA may provide a minimally invasive procedure for LCa subtyping, complementing standard diagnostic procedures.
\end{abstract}

Keywords: DNA Methylation; Lung Cancer; Subtyping; Cell-free DNA; Liquid Biopsy; Epigenetic Biomarker

\section{Introduction}

Lung cancer (LCa) is estimated to be the most commonly diagnosed cancer worldwide and the leading cause of cancer-related death in 2018 [1]. Most LCa cases are diagnosed at an advanced 
stage, endowing a modest five-year survival of $16 \%$, despite continuing improvements in diagnosis and treatment [2,3]. Smoking is the most well-established LCa risk factor, as $85 \%$ of LCa cases are attributable to cigarette carcinogens, including benzopyrenes [4]. Interestingly, a shift in LCa topography and dominant subtype has been observed over the last 50 years due to alterations in cigarette manufacturing [5]. Indeed, decreased nicotine leads to increased puff volume, which translates into higher proportions of peripheral versus centrally located tumors [6]. LCa is generally divided into two major subtypes: small-cell lung cancer (SCLC, about 15\% of all cases) and non-SCLC (NSCLC), which mostly comprises adenocarcinoma (AdC), squamous cell carcinoma (SCC), and large-cell carcinoma (LCC), as well as other less frequent histotypes [5]. Presently, AdC accounts for more than $40 \%$ of all LCa and has emerged as the main subtype because of the increasing access of tobacco smoke to the peripheral lung structures [5]. Although prognosis is strongly associated with stage, never-smokers and female patients usually endure better prognosis [7]. SCC constitutes, nowadays, the second most frequent LCa subtype, whereas SCLC is considered the most aggressive form of LCa (two-year survival rate of $10 \%$ ), and both are also associated with tobacco smoke [5].

LCa subtyping is essential in treatment decisions and prognosis [8]. Indeed, the introduction of targeted therapy for epidermal growth factor receptor (EGFR), anaplastic lymphoma kinase (ALK), and ROS proto-oncogene 1 (ROS1) mutations increased the importance of discriminating AdC from SCC, since those mutations are highly associated with the former [9]. Additionally, with the recent introduction of the immune checkpoint inhibitors in NSCLC treatment, LCa subtyping became a crucial process for LCa treatment [9]. LCa diagnosis is usually based on pathological assessment of tissue fragments or cells collected either by bronchoscopy, fine-needle aspiration, or core-needle biopsy, depending on the tumor's location and accessibility $[10,11]$. Nonetheless, in a sizeable proportion of cases, the material obtained is not sufficient for LCa subtyping [10], and the distinction between AdC and SCC may not be possible by morphology alone, even if assisted by immunohistochemistry [8]. Furthermore, these are invasive procedures amenable to complications such as hemorrhage and pneumothorax [8]. Molecular testing may provide higher specificity and decrease biopsy-associated risks. Indeed, an assay comprising the expression of eight microRNAS among four LCa subtypes (nonsquamous NSCLC, SCC, carcinoid, and SCLC) has shown high sensitivity and specificity, but it required tissue obtained from resections or biopsies and cytological samples, which might be difficult to obtain based on tumor location [12]. Thus, assays based on minimally invasive procedures are needed. Indeed, a study using a three-microRNA panel discriminated SCLC from NSCLC with high diagnostic accuracy in plasma samples [3].

LCa develops through a multistep process that includes altered DNA status [13]. Promoter hypermethylation of cancer-related genes is a common alteration, often associated with inactivation of tumor-suppressive genes [13], and its assessment has been proposed as a candidate biomarker for cancer detection and monitoring because of its stability and easy detection in tissue and body fluids [14,15]. Furthermore, the detection of circulating cell-free DNA (ccfDNA) methylation in plasma/serum samples may better represent tumor heterogeneity than tissue biopsy, being also less invasive [16]. Thus, we sought to evaluate the feasibility of using methylation of four gene promoters, previously characterized as hypermethylated in cancer [15-19], to discriminate among the major LCa subtypes in ccfDNA extracted from plasma, by means of quantitative methylation-specific PCR (qMSP). Selection of candidate genes APC, HOXA9, RAR $\beta 2$, and RASSF1A was based on published literature [17,20-22], including our previous experience [15], since methylation levels disclosed differences among LCa subtypes, suggesting a role as biomarkers.

\section{Materials and Methods}

\subsection{Patients and Sample Collection}

Three independent study groups of LCa patients were included in this study. Study group \#1 comprised 152 LCa patients diagnosed and treated at the Portuguese Oncology Institute of Porto (IPO 
Porto) between 2001 and 2016, from whom tissues were obtained from lung resection or tissue biopsy specimens. Study group \#2 included 129 LCa patients primarily diagnosed at IPO Porto, between 2015 and 2017, from whom blood samples were collected before any treatment. Study group \#3 comprised 28 plasma samples from patients suspect of harboring LCa but were found to carry benign lung disease, collected at IPO Porto between 2015 and 2019. Plasma was isolated from blood by centrifugation at $2000 \mathrm{rpm}$ for $10 \mathrm{~min}$ at $4{ }^{\circ} \mathrm{C}$, and subsequently frozen at $-80{ }^{\circ} \mathrm{C}$ until further use. Tissue samples were obtained for each patient in study group \#1, routinely fixed, and paraffin-embedded for standard pathological examination by H\&E and specific immunostaining for tumor classification, grading, and staging $[5,23,24]$. Relevant clinical data were collected from clinical charts, and a database was constructed for statistical analysis purposes. This study was approved by the institutional review board of IPO Porto (Comissão de Ética para a Saúde, CES 120/2015). All patients enrolled signed informed consent according to the Declaration of Helsinki ethical principles.

\subsection{DNA Extraction}

Regarding study group \#1, tumor areas were identified by an experienced pathologist in H\&E slides. Eight micrometer tissue sections were cut, and tumor areas were macrodissected to maximize the proportion of malignant cells (>70\%), deparaffinized and rehydrated using xylene and $100 \%$ ethanol, respectively, and digested with $60 \mu \mathrm{L}$ of proteinase $\mathrm{K}\left(20 \mathrm{mg} / \mathrm{mL}\right.$, Sigma-Aldrich ${ }^{\circledR}$, Schnelldorf, Germany). DNA was extracted using a standard phenol-chloroform protocol previously described [25]. Concerning study groups \#2 and \#3, ccfDNA was extracted from $2 \mathrm{~mL}$ of plasma using QIAmp MinElute ccfDNA (Qiagen, Hilden, Germany), according to the manufacturer's protocol and as previously described [15]. All extracted DNA was stored at $-20^{\circ} \mathrm{C}$ until further use.

\subsection{Sodium Bisulfite Modification, Whole Genome Amplification (WGA), and DNA Quantification}

Sodium bisulfite modification was performed using EZ DNA Methylation-Gold ${ }^{\mathrm{TM}}$ (Zymo Research, Orange, CA, USA) according to the manufacturer's recommendations. In tissue samples from study group \#1, sodium bisulfite converted DNA was eluted in $60 \mu \mathrm{L}$ of distilled water, whereas ccfDNA from study groups \#2 and \#3 were eluted in $10 \mu \mathrm{L}$ of distilled water. One microgram of CpGenome ${ }^{\mathrm{TM}}$ Universal Methylated DNA (Millipore, Temecula, CA, USA) was sodium bisulfite converted and used for control purposes. All sodium bisulfite converted DNA was stored at $-80^{\circ} \mathrm{C}$ until further use. WGA of ccfDNA extracted from study group \#2 and \#3 plasma samples was carried out using an EpiTect Whole Bisulfitome Kit (Qiagen, Hilden, Germany) according to the manufacturer's instructions and as previously described [15]. Tissue-extracted DNA was quantified by a NanoDrop Lite Spectrophotometer (NanoDrop Technologies, Wilmington, DE, USA). Extracted, sodium bisulfite converted, and amplified ccfDNA was quantified using a Qubit 2 Fluorometer (Invitrogen, Carlsbad, CA, USA) following the manufacturer's recommendations. The median ccfDNA concentration after extraction was $2.07 \mathrm{ng} / \mu \mathrm{L}$ (range: $0.392-26.6 \mathrm{ng} / \mu \mathrm{L}$ ) and after WGA was $70.6 \mathrm{ng} / \mu \mathrm{L}$ (range: 0.756 to $>120 \mathrm{ng} / \mu \mathrm{L}$ ) for study group \#2. Moreover, for study group \#3, the median ccfDNA concentration after extraction was $0.660 \mathrm{ng} / \mu \mathrm{L}$ (range: $0.132-3.36 \mathrm{ng} / \mu \mathrm{L}$ ) and after WGA was $60.3 \mathrm{ng} / \mu \mathrm{L}$ (range: $0.336-120 \mathrm{ng} / \mu \mathrm{L})$.

\subsection{Quantitative Methylation-Specific PCR ( $q M S P$ )}

QMSP was performed to assess APC, HOXA9, RAR $\beta 2$, and RASSF1A promoter methylation levels, and $\beta$-Actin served as a reference gene. For study group \#1, sodium bisulfite modified DNA was used as a template, and reactions were carried out in 384-well plates using a LightCycler 480 Instrument (Roche Diagnostics, Manheim, Germany). Briefly, per well, $2 \mu \mathrm{L}$ of modified DNA and $5 \mu \mathrm{L}$ of KAPA SYBRßFAST qPCR Master Mix (Kapa Biosystems, MA, USA) were used. The primers' volumes and conditions used for each gene are listed in Table S1. Because of ccfDNA quantity limitations, in study groups \#2 and \#3, APC, RASSF1A, and $\beta$-Actin were run in a multiplex qMSP reaction using TaqMan probes and Xpert Fast Probe (GRISP, Porto, Portugal), whereas RAR $\beta 2$ and HOXA9 were run in a 
separate qMSP reaction. Primers, probes, and fluorochromes used are listed in Table S2. Six microliters of amplified ccfDNA was used as template, and the multiplex qMSP assays were carried out using 96-well plates in a 7500 Sequence Detector (Applied Biosystems, Perkin Elmer, CA, USA). All samples were run in triplicate, and a maximum 0.38 deviation between replicates was used. Amplification cycles above 40 were considered a "no result", and these samples were not considered for further analysis.

Sodium bisulfite modified CpGenome ${ }^{\mathrm{TM}}$ Universal Methylated DNA was subjected to a series of dilutions ( $5 \times$ dilution factor) and used to generate a standard curve allowing DNA relative quantification and plate efficiency assessment. Efficiency values above $90 \%$ were considered in each plate. An efficiency difference of maximum $5 \%$ between plates was considered. Relative methylation levels were generated calculating the ratio between the methylation levels of each gene and the respective value of $\beta$-Actin and multiplied by 1000 for easier tabulation [26,27].

\subsection{Statistical Analysis}

Non-parametric tests were used for comparing the distribution of methylation levels among different LCa subtypes and to evaluate associations with clinicopathological parameters (primarily by Kruskal-Wallis tests for three or more groups, followed by pairwise comparisons using Mann-Whitney $\mathrm{U}$ tests and Bonferroni's correction, when applicable). The distribution of methylation levels was presented graphically in a scatter plot with a logarithmic scale. Zero values were transformed in value " 1 " for better representation and easier analysis [15]. A Spearman non-parametric test was performed to assess correlations between age and gene promoter methylation levels. Receiver operator characteristic (ROC) curve analysis was performed for each gene to assess biomarker performance. Samples were categorized as methylated (positive) or unmethylated (negative) based on the cutoff determined through ROC curve analysis, (i.e., the one providing the highest sensitivity and specificity, Youden's J index) [28]. Validity estimates (sensitivity, specificity, and accuracy) were also determined. For this, multiple analyses of ROC curves generated via resampling randomly and dividing the sample into training $(70 \%)$ and validation $(30 \%)$ sets was performed. The cutoff value was estimated in the training set, and the validity estimates were calculated in the validation set using that cutoff. This procedure was repeated 1000 times, and the mean of the sensitivities and specificities was calculated, as previously described $[15,29]$. Gene panels were defined as positive when at least one of the genes was categorized as methylated (positive). The multiple ROC curve analysis was performed using $\mathrm{R}$ v3.4.4. Two-tailed $p$ value calculations and ROC analysis were performed using a computer assisted program (SPSS Version 24.0, Chicago, IL, USA). Graphics were assembled with GraphPad 6 Prism (GraphPad Software, La Jolla, CA, USA).

\section{Results}

\subsection{Clinical and Pathological Data}

Relevant clinical and pathological data of study groups \#1 and \#2 are depicted in Table 1, whereas study group \#3 information is displayed in Table 2. No correlations were found between patients' age and gene promoter methylation levels in all study groups. Additionally, no differences were observed in the distribution of gender between study groups \#2 and \#3 ( $p=0.391)$.

Associations between clinical stage and gene methylation levels were found, namely in study group \#1 between stages I, II, and III; stage IV for APC, RAR $\beta 2$, and RASSF1A (Supplementary Materials, Table S3); and for RASSF1A between stages III and IV in study group \#2 (Supplementary Materials, Table S4). 
Table 1. Clinicopathological features of lung cancer (LCa) patients from study groups \#1 and \#2.

\begin{tabular}{ccc}
\hline \multirow{2}{*}{ Clinicopathological Features } & Study Group \#1 & Study Group \#2 \\
\cline { 2 - 3 } & Tissue Samples & Plasma Samples \\
\hline Patients (n) & 152 & 129 \\
\hline Gender & 113 & 91 \\
Male (M) & 39 & 38 \\
Female (F) & $65(45-83)$ & $66(38-89)$ \\
\hline Age, median (range) & & $65(35 \mathrm{M} ; 30 \mathrm{~F}) ; 50 \%$ \\
\hline Histological Subtype, $\mathbf{n} ; \mathbf{\%} \mathbf{1}$ & $56(26 \mathrm{M} ; 30 \mathrm{~F}) ; 39 \%$ & $42(39 \mathrm{M} ; 3 \mathrm{~F}) ; 31 \%$ \\
AdC & $65(62 \mathrm{M} ; 3 \mathrm{~F}) ; 41 \%$ & - \\
SCC & $6(6 \mathrm{M}) ; 4 \%$ & $19(16 \mathrm{M} ; 3 \mathrm{~F}) ; 15 \%$ \\
LCC & $25(19 \mathrm{M} ; 6 \mathrm{~F}) ; 16 \%$ & \\
SCLC & &
\end{tabular}

\section{Clinical stage}

$\begin{array}{lll}\text { I } & 74 & 15 \\ \text { II } & 33 & 11 \\ \text { III } & 24 & 27 \\ \text { IV } & 21 & 76\end{array}$

${ }^{1}$ Includes 3 non-small-cell lung carcinoma (NSCLC) in study group \#2. Abbreviations: AdC, adenocarcinoma; F, female; LCC, large-cell carcinoma; m, Male; SCLC, small-cell lung cancer; SCC, squamous cell carcinoma; n.a., not available.

Table 2. Clinicopathological features of benign lung disease patients from study group \#3.

\begin{tabular}{cc}
\hline \multirow{2}{*}{ Clinicopathological Features } & Study Group \#3 \\
\cline { 2 - 2 } & Benign Lung Diseases Plasma Samples \\
\hline Patients (n) & 28 \\
\hline Gender & 22 \\
Male (M) & 6 \\
Female (F) & $63(40-86)$ \\
\hline Age, median (range) & 9 \\
Benign lung disease & 3 \\
Inflammatory processes & 4 \\
Tuberculosis & 1 \\
Chondroid hamartoma & 1 \\
Silicosis & 10 \\
Fibrosis &
\end{tabular}

\subsection{Distribution of Gene Promoter Methylation Levels in ccfDNA}

$A P C, H O X A 9, R A R \beta 2$, and RASSF1A promoter methylation levels were evaluated in plasma samples from benign lung disease and LCa patients to assess whether they might differ among malignant and benign lung disease (Figure 1). APC and RASSF1A displayed higher methylation levels in LCa compared to benign cases ( $p=0.006$ and $p=0.033$, respectively) (Figure 1 ), whereas HOXA9 and $R A R \beta 2$ did not disclose significant differences ( $p=0.329$ and $p=0.133$, respectively) (Figure 1 ). 

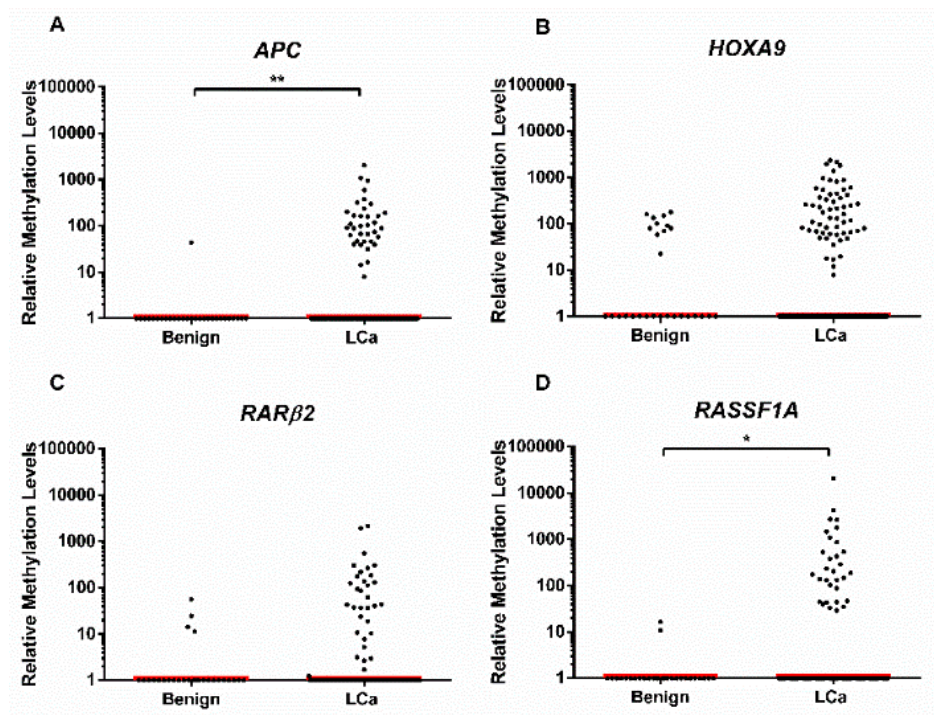

Figure 1. Scatter plot of (A) $A P C$ (number of values that fall in the $x$ axis: benign $=27, \mathrm{LCa}=93$ ), (B) HOXA9 (number of values that fall in the $x$ axis: benign $=17, \mathrm{LCa}=71$ ), (C) RAR $\beta 2$ (number of values that fall in the $x$ axis benign $=24, \mathrm{LCa}=92$ ), and (D) RASSF1A (number of values that fall in the $x$ axis: benign $=26, \mathrm{LCa}=98$ ); methylation level distribution among lung cancer (LCa) patients from study group \#2 (plasma samples) and benign lung diseases patients from study group \#3 (plasma samples). Mann-Whitney U test. ${ }^{*} p<0.05,{ }^{* *} p<0.01$. Red horizontal line represents the median methylation level.

Because LCa plasma samples displayed higher APC and RASSF1A methylation levels than benign lung disease cases in ccfDNA, their performance for LCa detection was assessed individually and in panel (Table 3, Supplementary Material, Figure S1). The panel comprising both genes disclosed $38.2 \%$ sensitivity and $92.8 \%$ specificity, corresponding to an overall accuracy of $47.6 \%$ for LCa detection (Table 3).

Table 3. Biomarker performance of promoter gene methylation for LCa detection in plasma samples.

\begin{tabular}{ccccc}
\hline Genes & AUC $^{\mathbf{1}}$ & Sensitivity $\%$ & Specificity $\%$ & Accuracy $\%$ \\
\hline$A P C$ & 0.622 & 25.0 & 96.4 & 37.3 \\
$R A S S F 1 A$ & 0.591 & 23.7 & 95.4 & 36.0 \\
$A P C / R A S S F 1 A$ & - & 38.2 & 92.8 & 47.6 \\
\hline
\end{tabular}

${ }^{1}$ AUC-area under the curve.

\subsection{Gene Promoter Methylation Level Distribution Among Major LCa Subtypes}

In study group \#1, SCLC disclosed significantly higher APC, HOXA9, RAR $\beta 2$, and RASSF1A promoter methylation levels compared to NSCLC ( $p<0.0001$ for all genes, except for HOXA9, $p=0.021)$ (Figure 2A), whereas in ccfDNA (study group \#2), only HOXA9 and RASSF1A retained significant statistical differences ( $p<0.0001$ for both genes) (Figure 2B). 
A
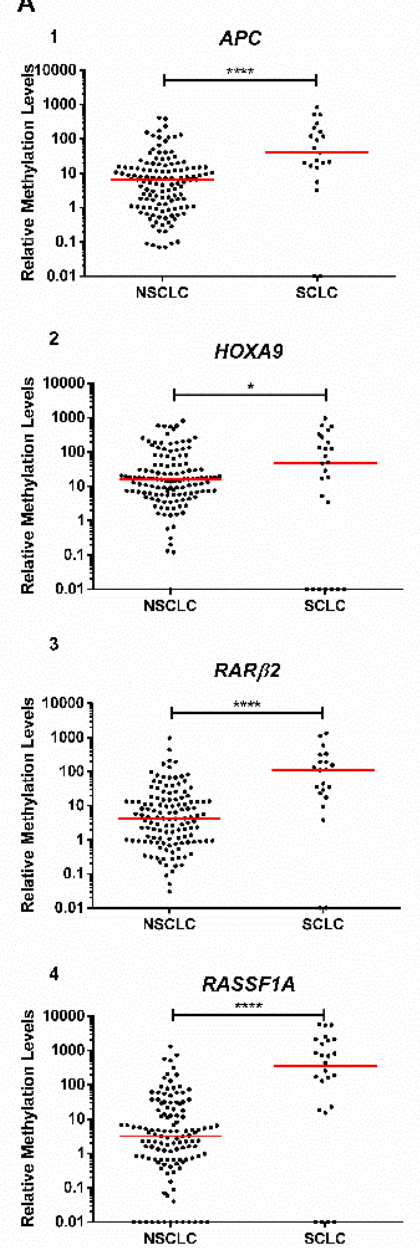
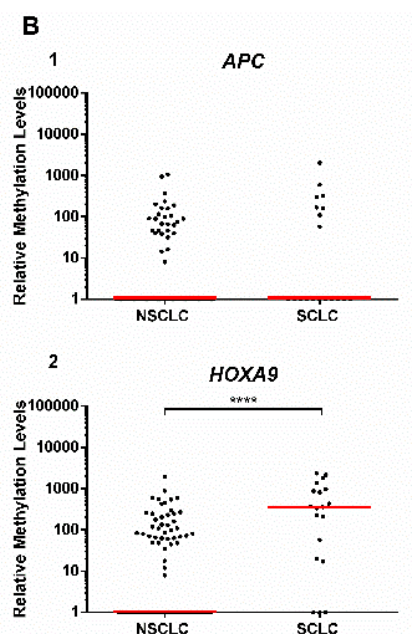

3
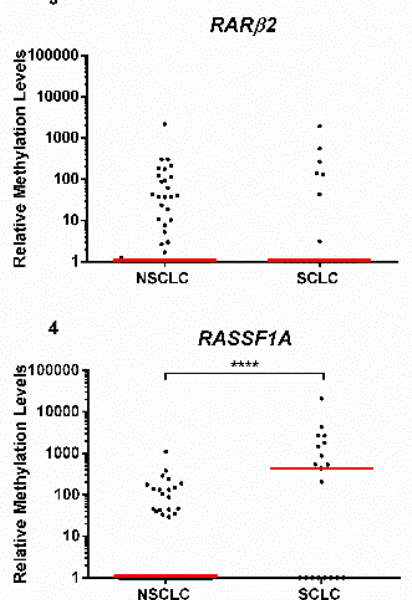

Figure 2. Scatter plot of (A) (1) APC, (2) HOXA9, (3) RAR 32 , and (4) RASSF1A methylation level distributions among major lung cancer (LCa) subtypes (non-small-cell lung cancer (NSCLC) and small-cell lung cancer (SCLC)) in study group \#1 (tissue samples) and (B) (1) APC (number of values that fall in the $x$ axis: NSCLC $=82$, SCLC $=11$ ), (2) HOXA9 (number of values that fall in the $x$ axis: NSCLC $=68$, SCLC $=3$ ), (3) RAR 32 (number of values that fall in the $x$ axis: NSCLC $=84$, SCLC $=12$ ), and (4) RASSF1A (number of values that fall in the $x$ axis: NSCLC $=90$, SCLC $=8$ ) among NSCLC and SCLC in study group \#2 (plasma samples). Mann-Whitney U test. ${ }^{*} p<0.05$ and ${ }^{* * * *} p<0.0001$. Red horizontal line represents the median methylation level.

\subsection{Biomarker Performance for SCLC Detection in Liquid Biopsies}

Since HOXA9 and RASSF1A methylation levels were higher in SCLC in comparison with NSCLC in ccfDNA (study group \#2), the performance of these genes for SCLC identification was evaluated (Table 4, Supplementary Material, Figure S2). HOXA9 detected SCLC with 64\% sensitivity, whereas RASSF1A individually disclosed $96 \%$ specificity (Table 4 ).

Table 4. Biomarker performance of promoter gene methylation for SCLC detection in study group \#2 (plasma samples).

\begin{tabular}{ccccc}
\hline Genes & AUC $^{\mathbf{1}}$ & Sensitivity $\%$ & Specificity $\%$ & Accuracy $\%$ \\
\hline HOXA9 & 0.805 & 63.9 & 84.2 & 82.2 \\
RASSF1A & 0.747 & 52.0 & 96.2 & 79.1 \\
\hline
\end{tabular}

${ }^{1}$ AUC-area under the curve. 


\subsection{Gene Promoter Methylation Levels According to LCa Histological Subtypes}

In study group \#1, statistically significant differences among AdC, SCC, and SCLC were depicted for APC, HOXA9, RAR $\beta 2$, and RASSF1A promoter methylation levels ( $p<0.001$ for all genes) (Figure $3 \mathrm{~A}$ ). In detail, $A P C, R A R \beta 2$, and RASSF1A showed statistically different methylation levels between AdC, SCC and SCLC $(p<0.0001)$ (Figure 3A), whereas HOXA9 methylation levels were higher in SCC in comparison with AdC $(p<0.001)$ (Figure 3A). In ccfDNA (study group \#2), promoter methylation levels of HOXA9 and RASSF1A were higher in SCLC in comparison with AdC and SCC $(p<0.001)$ (Figure 3B).
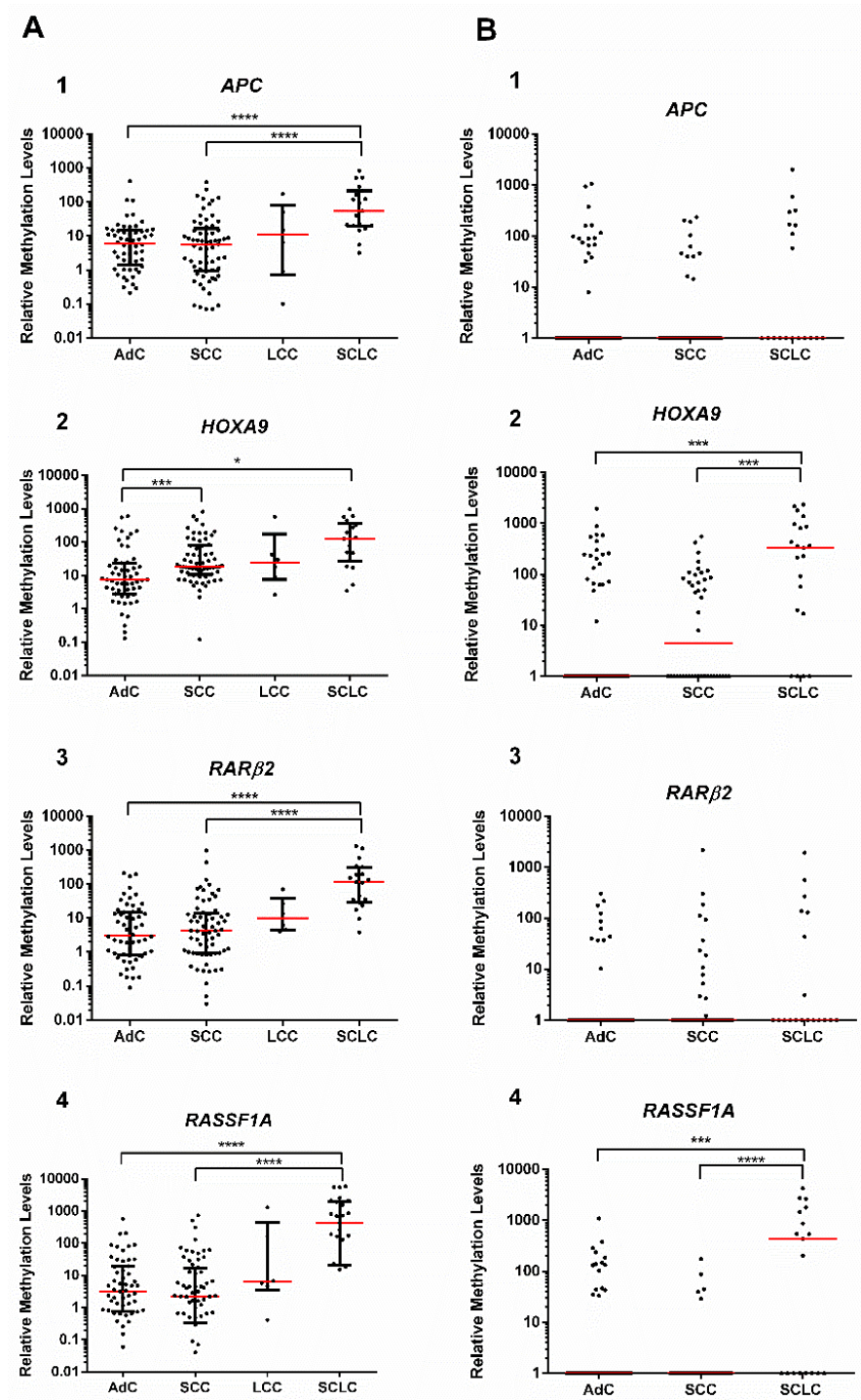

Figure 3. Scatter plot of (A) (1) APC, (2) HOXA9, (3) RAR 2 , and (4) RASSF1A methylation level distributions among lung cancer (LCa) histological subtypes (adenocarcinoma (AdC), squamous cell carcinoma (SCC), large cell carcinoma (LCC), and small-cell lung cancer (SCLC)) in study group \#1 (tissue samples) and (B) (1) APC (number of values that fall in the $x$ axis: $\mathrm{AdC}=48, \mathrm{SCC}=31, \mathrm{SCLC}=11$ ), (2) HOXA9 (number of values that fall in the $x$ axis: $\mathrm{AdC}=44, \mathrm{SCC}=21, \mathrm{SCLC}=3$ ), (3) RAR 32 (number of values that fall in the $x$ axis: $\mathrm{AdC}=54, \mathrm{SCC}=27, \mathrm{SCLC}=12$ ), and (4) RASSF1A (number of values that fall in the $x$ axis: $\mathrm{AdC}=50, \mathrm{SCC}=37, \mathrm{SCLC}=8$ ) methylation level distributions among LCa histological subtypes (AdC, SCC, and SCLC) in study group \#2 (plasma samples). Kruskal-Wallis test, followed by pairwise comparison though Mann-Whitney $U$ and Bonferroni's correction. ${ }^{*} p<0.05$, ${ }^{* *} p<0.01,{ }^{* * *} p<0.001$, and ${ }^{* * * *} p<0.0001$. Red horizontal line represents the median methylation level. 
Because HOXA9 methylation levels were significantly different between AdC and SCC in tissue samples, their biomarker performance for SCC detection in ccfDNA was evaluated. HOXA9 hypermethylation detected SCC with $55.2 \%$ sensitivity, $74.3 \%$ specificity, and $71.6 \%$ accuracy, corresponding to an area under the curve (AUC) of 0.657 (Table 5, Supplementary Material, Figure S3).

Table 5. Biomarker performance of HOXA9 promoter gene methylation for SCC detection in study group \#1 (tissue samples).

\begin{tabular}{cc}
\hline Validity Estimates & HOXA9 Methylation \\
\hline Sensitivity \% & 55.2 \\
Specificity \% & 74.3 \\
Accuracy \% & 71.6 \\
AUC & 0.657 \\
\hline
\end{tabular}

\section{Discussion}

Currently, LCa screening is only recommended for high-risk smokers; thus, most LCa cases are diagnosed at an advanced stage, entailing poor survival [2,30]. After LCa suspicion by imaging techniques, tissue biopsy and/or cytology are performed to confirm diagnosis and determine the LCa subtype [10]. Frequently, only a limited amount of material is obtained, which may result in the impossibility to perform a diagnosis or to adequately subtype the tumor, eventually leading to re-biopsy [31]. With the introduction of targeted therapies, a renewed interest in LCa subtyping emerged. In the presence of a positive biopsy, the LCa subtype can be determined by morphology in combination with immunohistochemistry [5]. Markers such as TTF1 for AdC and p40 for SCC are currently used for NSCLC subtyping [5,32]. Nevertheless, about $10 \%$ of NSCLC are rendered as "not otherwise specified" [5]. This may impact treatment decisions and patient prognoses, since AdC patients may benefit from targeted therapies for EGFR and ROS1 or ALK mutations that significantly increase patient survival [33]. Additionally, therapy with pemetrexed or bevacizumab is contraindicated for SCC patients because of frequent complications $[34,35]$. Thus, correct LCa subtyping is critical to improve patient prognosis through a better selection of therapeutic strategies. Aberrant promoter methylation of cancer-related genes is a frequent and early alteration in carcinogenesis and may be easily detected in tissue and body fluids, constituting a potential biomarker for cancer detection and monitoring $[15,26,36]$. Hence, we aimed to determine whether promoter methylation of selected genes might allow for accurate discrimination among LCa subtypes in ccfDNA samples, providing a minimally invasive ancillary tool.

In tissue samples, higher methylation levels for all genes were found in SCLC compared to NSCLC, which is in line with previous reports on high RASSF1A and RAR $\beta 2$ methylation levels in SCLC cell lines and tissue samples [22,37-40]. Concerning APC promoter methylation, however, our results contradict previous studies that showed higher levels in NSCLC $[39,41]$. This discrepancy may be due to methodological differences, since conventional methylation-specific PCR (MSP), a qualitative analysis, was used in those studies, whereas we employed qMSP, a quantitative method with higher sensitivity and specificity [42]. High HOXA9 methylation levels were previously reported in NSCLC [19-21,27], but to the best of our knowledge, this is the first study demonstrating higher HOXA9 methylation levels in SCLC tissue and ccfDNA samples. Moreover, higher HOXA9 methylation levels were found in SCC compared to AdC in tissue samples, confirming previous studies [43,44].

Concerning ccfDNA samples, APC, RASSF1A, and HOXA9 were found hypermethylated in SCLC. In a previous study of ours, $A P C$ and $R A R \beta 2$ displayed higher methylation levels in SCLC compared to NSCLC in women [15]. The inclusion of both genders and a larger sample size of SCLC may explain these dissimilarities. Moreover, the higher RASSF1A methylation levels in ccfDNA samples from SCLC compared to NSCLC patients are in accordance with a previous study [45]. The differences in LCa stage distribution between study group \#1 and study group \#2 mainly are due to the nature of the samples, since study group \#1 comprises tissue samples obtained mostly from surgical specimens, 
thus corresponding to earlier LCa stages. Furthermore, a small study group of patients with benign lung diseases was also included in this study. These patients had suspicious alterations detected in imaging exams that, consequently, were subjected to tissue biopsy, and LCa was not confirmed. Comparatively, LCa patients displayed higher methylation levels of APC and RASSF1A than those patients with benign lung disease, indicating that LCa detection may be achieved by analyzing DNA methylation in ccfDNA liquid biopsies, as previously demonstrated [15,17,27], complementing other diagnostic modalities (Figure 4). Nonetheless, these results need to be further validated in order to increase the assay sensitivity and evaluate their usefulness in clinical practice.

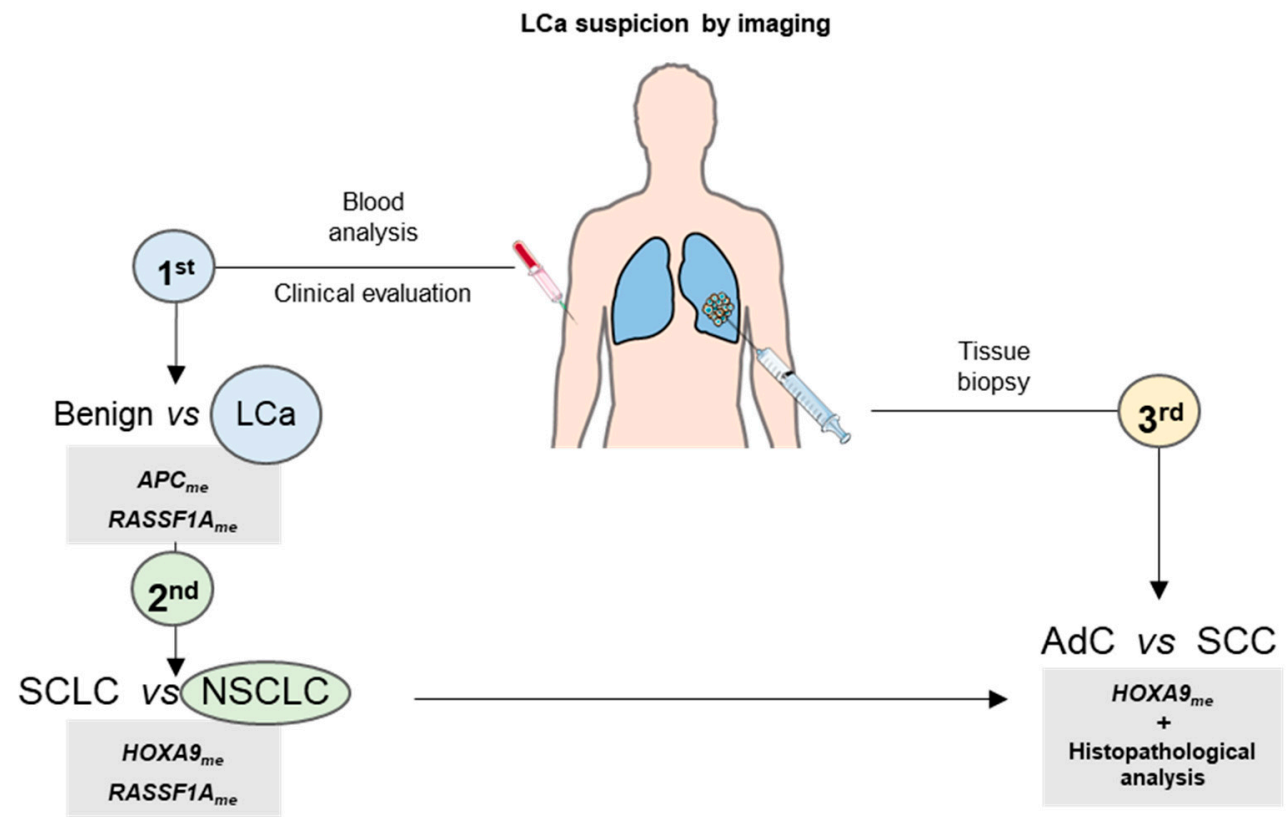

Figure 4. Proposed algorithm for lung cancer (LCa) subtyping when LCa is suspected by imaging. Firstly, blood analysis by assessing APC and RASSF1A methylation levels could be performed in combination with clinical evaluation in order to detect LCa. Then, the methylation levels of HOXA9 or RASSF1A could be evaluated to aid in determining the major LCa subtype present (non-small-cell lung cancer (NSCLC) vs small-cell lung cancer (SCLC)). After, if NSCLC present, a tissue biopsy could be performed, and HOXA9 methylation levels could aid histopathological analysis in discriminating between adenocarcinoma (AdC) and squamous cell carcinoma (SCC).

Several studies using microRNA (miRNA) panels attempted to discriminate among the different LCa subtypes in histological and cytological samples [12,46,47]. One of these panels, miRview ${ }^{\circledR}$ lung (Rosetta Genomics), is based on the expression of eight miRNAs and displayed over $90 \%$ sensitivity for SCLC detection in tissue samples [12]. In our study, HOXA9 displayed $64 \%$ sensitivity, whereas RASSF1A showed $96 \%$ specificity for SCLC detection in ccfDNA liquid biopsies. SCLC diagnosis using liquid biopsies in combination with clinical evaluation could allow for faster diagnosis and, consequently, sooner therapeutic decision, eventually precluding the need for an invasive procedure (i.e., biopsy).

Additionally, DNA methylation assessment in ccfDNA might aid in the diagnosis of tumors not assessable by the currently used techniques or in cases of limited diagnostic material, which do not allow for accurate diagnosis. To the best of our knowledge, this is the first study reporting a test assessing HOXA9 and RASSF1A methylation levels for SCLC detection in liquid biopsies. In combination with clinical evaluation, including imaging and smoking status, this test may assist in LCa diagnosis (Figure 4). Thus, ccfDNA testing might be used as first line assay in the investigation of LCa suspects in combination with other diagnostic approaches, and a tissue biopsy would be performed only when it was negative for SCLC. In this scenario, NSCLC is likely to be present, and, as above mentioned, the 
differential diagnosis between AdC and SCC is not always possible (Figure 4). Interestingly, higher HOXA9 methylation levels were depicted in SCC compared to AdC, although with lower sensitivity and specificity compared to miRview ${ }^{\circledR}$ lung [12]. However, since we only analyzed one gene promoter, we are tempted to speculate whether our assay would be a faster and less expensive method for discriminating AdC from SCC. Nevertheless, further studies are required to assess its usefulness in a "real-world" scenario.

The main limitations of our study include the relatively small number of benign lung diseases and SCLC samples as well as the exclusion of other tumors with neuroendocrine differentiation such as carcinoids and large-cell neuroendocrine carcinoma. Nonetheless, this is an innovative proof-of-concept study that proposes LCa subtyping in a single blood analysis alongside clinical evaluation, complementing invasive tests to assist in patient diagnosis.

\section{Conclusions}

We report a methylation-based test for LCa subtyping in liquid biopsies. Such tests might not only speed up the diagnostic process, but they could also aid in clinical decision making. The clinical usefulness of these preliminary results requires further validation in larger independent cohorts.

Supplementary Materials: The following are available online at http://www.mdpi.com/2077-0383/8/9/1500/s1, Supplementary tables: Table S1: Primers used in study group \#1 tissue samples and qMSP conditions for each tested gene, Table S2: Primers and probe sequences used in study group \#2 and \#3 plasma samples with respective fluorochrome and quencher. Table S3: Associations between clinical stage and APC, HOXA9, RAR $\beta 2$, and RASSF1A methylation levels in study group \#1. $P$ values obtained by Kruskal-Wallis followed by Mann-Whitney $\mathrm{U}$ tests and Bonferroni's correction. Table S4: Associations between clinical stage and APC, HOXA9, RAR $\beta 2$, and RASSF1A methylation levels in study group \#2. P values obtained by Kruskal-Wallis followed by Mann-Whitney U tests and Bonferroni's correction. Supplementary figures: Figure S1. Receiver operating characteristic (ROC) curve of (A) APC and (B) RASSF1A for LCa detection in plasma samples (study group \#2 and study group \#3). Figure S2. Receiver operating characteristic (ROC) curve of (A) HOXA9 and (B) RASSF1A for small-cell lung cancer (SCLC) detection in plasma samples (study group \#2). Figure S3. Receiver operating characteristic (ROC) curve of HOXA9 for squamous cell carcinoma detection in tissue samples (study group \#1).

Author Contributions: S.P.N. and F.D. performed DNA extraction, qMSP, analyzed the data, and drafted the manuscript. C.M.-B. and V.C. assisted in laboratorial procedures and data analyses. A.V.S. collected follow-up data. J.O. and M.S. assisted in patient enrollment for sample collection. S.P. and A.L.C. assisted in histological techniques. J.R. and L.A. assisted in statistical analyses. R.H. and C.J. designed and supervised the study and revised the manuscript. All authors read and approved the final manuscript.

Funding: This work was supported by a grant from Research Center of Portuguese Oncology Institute of Porto (PI 74-CI-IPOP-19-2016). C.M.-B. was supported by was supported by Núcleo Regional da Madeira da Liga Portuguesa Contra o Cancro \& Diário de Notícias.

Acknowledgments: We acknowledge all the patients that accepted to participate in this study. We thank the Nursing Staff from Department of Laboratory Medicine, and Berta Reis in particular, and the staff at Lung Cancer Clinic of Portuguese Oncology Institute of Porto for their participation in patient enrollment and sample collection. This study would not be possible without them.

Conflicts of Interest: The authors declare no conflicts of interest.

\section{References}

1. Bray, F.; Ferlay, J.; Soerjomataram, I.; Siegel, R.L.; Torre, L.A.; Jemal, A. Global cancer statistics 2018: GLOBOCAN estimates of incidence and mortality worldwide for 36 cancers in 185 countries. CA Cancer J. Clin. 2018. [CrossRef] [PubMed]

2. Lu, C.; Soria, J.C.; Tang, X.; Xu, X.C.; Wang, L.; Mao, L.; Lotan, R.; Kemp, B.; Bekele, B.N.; Feng, L.; et al. Prognostic factors in resected stage I non-small-cell lung cancer: A multivariate analysis of six molecular markers. J. Clin. Oncol. 2004, 22, 4575-4583. [CrossRef] [PubMed]

3. Lu, S.; Kong, H.; Hou, Y.; Ge, D.; Huang, W.; Ou, J.; Yang, D.; Zhang, L.; Wu, G.; Song, Y.; et al. Two plasma microRNA panels for diagnosis and subtype discrimination of lung cancer. Lung Cancer 2018, 123, 44-51. [CrossRef] [PubMed]

4. de Groot, P.; Munden, R.F. Lung cancer epidemiology, risk factors, and prevention. Radiol. Clin. N. Am. 2012, 50, 863-876. [CrossRef] [PubMed] 
5. William, D.; Travis, E.; Allen, P.; Alexander, M.; Andrew, G. WHO Classification of Tumors of the Lung, Pleura, Thymus and Heart Ch. 1; International Agency for Research on Cancer: Lyon, France, 2015; pp. 9-152.

6. McLean, A.E.B.; Barnes, D.J.; Troy, L.K. Diagnosing Lung Cancer: The Complexities of Obtaining a Tissue Diagnosis in the Era of Minimally Invasive and Personalised Medicine. J. Clin. Med. 2018, 7, 163. [CrossRef] [PubMed]

7. Pallis, A.G.; Syrigos, K.N. Lung cancer in never smokers: Disease characteristics and risk factors. Crit. Rev. Oncol. Hematol. 2013, 88, 494-503. [CrossRef] [PubMed]

8. Ofiara, L.M.; Navasakulpong, A.; Beaudoin, S.; Gonzalez, A.V. Optimizing tissue sampling for the diagnosis, subtyping, and molecular analysis of lung cancer. Front. Oncol. 2014, 4, 253. [CrossRef] [PubMed]

9. Reck, M.; Rabe, K.F. Precision diagnosis and treatment for advanced non-small-cell lung cancer. N. Engl. J. Med. 2017, 377, 849-861. [CrossRef]

10. Postmus, P.E.; Kerr, K.M.; Oudkerk, M.; Senan, S.; Waller, D.A.; Vansteenkiste, J.; Escriu, C.; Peters, S. Early and locally advanced non-small-cell lung cancer (NSCLC): ESMO Clinical Practice Guidelines for diagnosis, treatment and follow-up. Ann. Oncol. 2017, 28, iv1-iv21. [CrossRef]

11. Yao, X.; Gomes, M.M.; Tsao, M.S.; Allen, C.J.; Geddie, W.; Sekhon, H. Fine-needle aspiration biopsy versus core-needle biopsy in diagnosing lung cancer: A systematic review. Curr. Oncol. (Tor. Ont.) 2012, 19, e16-e27. [CrossRef]

12. Gilad, S.; Lithwick-Yanai, G.; Barshack, I.; Benjamin, S.; Krivitsky, I.; Edmonston, T.B.; Bibbo, M.; Thurm, C.; Horowitz, L.; Huang, Y.; et al. Classification of the four main types of lung cancer using a microRNA-based diagnostic assay. J. Mol. Diagn. 2012, 14, 510-517. [CrossRef] [PubMed]

13. Baylin, S.B.; Jones, P.A. Epigenetic determinants of cancer. Cold. Spring. Harb. Perspect. Biol. 2016, a019505. [CrossRef] [PubMed]

14. Costa-Pinheiro, P.; Montezuma, D.; Henrique, R.; Jeronimo, C. Diagnostic and prognostic epigenetic biomarkers in cancer. Epigenomics 2015, 7, 1003-1015. [CrossRef] [PubMed]

15. Nunes, S.P.; Moreira-Barbosa, C.; Salta, S.; Palma de Sousa, S.; Pousa, I.; Oliveira, J.; Soares, M.; Rego, L.; Dias, T.; Rodrigues, J.; et al. Cell-Free DNA Methylation of Selected Genes Allows for Early Detection of the Major Cancers in Women. Cancers (Basel) 2018, 10, 357. [CrossRef] [PubMed]

16. Han, X.; Wang, J.; Sun, Y. Circulating tumor DNA as biomarkers for cancer detection. Genom. Proteom. Bioinform. 2017, 15, 59-72. [CrossRef] [PubMed]

17. Begum, S.; Brait, M.; Dasgupta, S.; Ostrow, K.L.; Zahurak, M.; Carvalho, A.L.; Califano, J.A.; Goodman, S.N.; Westra, W.H.; Hoque, M.O.; et al. An epigenetic marker panel for detection of lung cancer using cell-free serum DNA. Clin. Cancer Res. 2011, 17, 4494-4503. [CrossRef] [PubMed]

18. Hsu, H.S.; Chen, T.P.; Hung, C.H.; Wen, C.K.; Lin, R.K.; Lee, H.C.; Wang, Y.C. Characterization of a multiple epigenetic marker panel for lung cancer detection and risk assessment in plasma. Cancer 2007, 110, 2019-2026. [CrossRef] [PubMed]

19. Liu, F.; Zhang, H.; Lu, S.; Wu, Z.; Zhou, L.; Cheng, Z.; Bai, Y.; Zhao, J.; Zhang, Q.; Mao, H. Quantitative assessment of gene promoter methylation in non-small cell lung cancer using methylation-sensitive high-resolution melting. Oncol. Lett. 2018, 15, 7639-7648. [CrossRef] [PubMed]

20. Wrangle, J.; Machida, E.O.; Danilova, L.; Hulbert, A.; Franco, N.; Zhang, W.; Glockner, S.C.; Tessema, M.; Van Neste, L.; Easwaran, H.; et al. Functional identification of cancer-specific methylation of CDO1, HOXA9, and TAC1 for the diagnosis of lung cancer. Clin. Cancer Res. 2014, 20, 1856-1864. [CrossRef] [PubMed]

21. Ooki, A.; Maleki, Z.; Tsay, J.-C.J.; Goparaju, C.; Brait, M.; Turaga, N.; Nam, H.-S.; Rom, W.N.; Pass, H.I.; Sidransky, D.; et al. A Panel of Novel Detection and Prognostic Methylated DNA Markers in Primary Non-Small Cell Lung Cancer and Serum DNA. Clin. Cancer Res. 2017, 23, 7141-7152. [CrossRef] [PubMed]

22. Burbee, D.G.; Forgacs, E.; Zochbauer-Muller, S.; Shivakumar, L.; Fong, K.; Gao, B.; Randle, D.; Kondo, M.; Virmani, A.; Bader, S.; et al. Epigenetic inactivation of RASSF1A in lung and breast cancers and malignant phenotype suppression. J. Natl. Cancer Inst. 2001, 93, 691-699. [CrossRef] [PubMed]

23. Amin, M.B. AJCC Cancer Staging Manual, 8th ed.; Springer: Berlin/Heidelberg, Germany, 2017.

24. Montezuma, D.; Azevedo, R.; Lopes, P.; Vieira, R.; Cunha, A.L.; Henrique, R. A panel of four immunohistochemical markers (CK7, CK20, TTF-1, and p63) allows accurate diagnosis of primary and metastatic lung carcinoma on biopsy specimens. Virchows Arch. 2013, 463, 749-754. [CrossRef] [PubMed] 
25. Monteiro-Reis, S.; Leca, L.; Almeida, M.; Antunes, L.; Monteiro, P.; Dias, P.C.; Morais, A.; Oliveira, J.; Henrique, R.; Jeronimo, C. Accurate detection of upper tract urothelial carcinoma in tissue and urine by means of quantitative GDF15, TMEFF2 and VIM promoter methylation. Eur. J. Cancer 2014, 50, $226-233$. [CrossRef] [PubMed]

26. Hoque, M.O.; Feng, Q.; Toure, P.; Dem, A.; Critchlow, C.W.; Hawes, S.E.; Wood, T.; Jeronimo, C.; Rosenbaum, E.; Stern, J.; et al. Detection of aberrant methylation of four genes in plasma DNA for the detection of breast cancer. J. Clin. Oncol. 2006, 24, 4262-4269. [CrossRef] [PubMed]

27. Hulbert, A.; Jusue-Torres, I.; Stark, A.; Chen, C.; Rodgers, K.; Lee, B.; Griffin, C.; Yang, A.; Huang, P.; Wrangle, J.; et al. Early Detection of Lung Cancer Using DNA Promoter Hypermethylation in Plasma and Sputum. Clin. Cancer Res. 2017, 23, 1998-2005. [CrossRef] [PubMed]

28. Schisterman, E.F.; Perkins, N.J.; Liu, A.; Bondell, H. Optimal cut-point and its corresponding Youden Index to discriminate individuals using pooled blood samples. Epidemiology 2005, 16, 73-81. [CrossRef] [PubMed]

29. Baker, S.G.; Kramer, B.S. Identifying genes that contribute most to good classification in microarrays. BMC Bioinform. 2006, 7, 407. [CrossRef]

30. Gouvinhas, C.; De Mello, R.A.; Oliveira, D.; Castro-Lopes, J.M.; Castelo-Branco, P.; Dos Santos, R.S.; Hespanhol, V.; Pozza, D.H. Lung cancer: A brief review of epidemiology and screening. Future Oncol. 2018. [CrossRef]

31. Field, R.W.; Smith, B.J.; Platz, C.E.; Robinson, R.A.; Neuberger, J.S.; Brus, C.P.; Lynch, C.F. Lung cancer histologic type in the surveillance, epidemiology, and end results registry versus independent review. J. Natl. Cancer Inst. 2004, 96, 1105-1107. [CrossRef]

32. Affandi, K.A.; Tizen, N.M.S.; Mustangin, M.; Zin, R. p40 Immunohistochemistry Is an Excellent Marker in Primary Lung Squamous Cell Carcinoma. J. Pathol. Transl. Med. 2018, 52, 283-289. [CrossRef]

33. Wu, Y.L.; Zhou, C.; Liam, C.K.; Wu, G.; Liu, X.; Zhong, Z.; Lu, S.; Cheng, Y.; Han, B.; Chen, L.; et al. First-line erlotinib versus gemcitabine/cisplatin in patients with advanced EGFR mutation-positive non-small-cell lung cancer: Analyses from the phase III, randomized, open-label, ENSURE study. Ann. Oncol. 2015, 26, 1883-1889. [CrossRef] [PubMed]

34. Hellmann, M.D.; Chaft, J.E.; Rusch, V.; Ginsberg, M.S.; Finley, D.J.; Kris, M.G.; Price, K.A.; Azzoli, C.G.; Fury, M.G.; Riely, G.J.; et al. Risk of hemoptysis in patients with resected squamous cell and other high-risk lung cancers treated with adjuvant bevacizumab. Cancer Chemother. Pharm. 2013, 72, 453-461. [CrossRef] [PubMed]

35. Fuld, A.D.; Dragnev, K.H.; Rigas, J.R. Pemetrexed in advanced non-small-cell lung cancer. Expert Opin. Pharm. 2010, 11, 1387-1402. [CrossRef] [PubMed]

36. Kneip, C.; Schmidt, B.; Seegebarth, A.; Weickmann, S.; Fleischhacker, M.; Liebenberg, V.; Field, J.K.; Dietrich, D. SHOX2 DNA methylation is a biomarker for the diagnosis of lung cancer in plasma. J. Thorac. Oncol. 2011, 6, 1632-1638. [CrossRef] [PubMed]

37. Walter, R.F.H.; Rozynek, P.; Casjens, S.; Werner, R.; Mairinger, F.D.; Speel, E.J.M.; Zur Hausen, A.; Meier, S.; Wohlschlaeger, J.; Theegarten, D.; et al. Methylation of L1RE1, RARB, and RASSF1 function as possible biomarkers for the differential diagnosis of lung cancer. PLOS ONE 2018, 13, e0195716. [CrossRef] [PubMed]

38. Yanagawa, N.; Tamura, G.; Oizumi, H.; Kanauchi, N.; Endoh, M.; Sadahiro, M.; Motoyama, T. Promoter hypermethylation of RASSF1A and RUNX3 genes as an independent prognostic prediction marker in surgically resected non-small cell lung cancers. Lung Cancer 2007, 58, 131-138. [CrossRef]

39. Toyooka, S.; Toyooka, K.O.; Maruyama, R.; Virmani, A.K.; Girard, L.; Miyajima, K.; Harada, K.; Ariyoshi, Y.; Takahashi, T.; Sugio, K.; et al. DNA methylation profiles of lung tumors. Mol. Cancer 2001, 1, 61-67.

40. Virmani, A.K.; Rathi, A.; Zöchbauer-Müller, S.; Sacchi, N.; Fukuyama, Y.; Bryant, D.; Maitra, A.; Heda, S.; Fong, K.M.; Thunnissen, F. Promoter methylation and silencing of the retinoic acid receptor- $\beta$ gene in lung carcinomas. J. Natl. Cancer Inst. 2000, 92, 1303-1307. [CrossRef]

41. Virmani, A.K.; Rathi, A.; Sathyanarayana, U.G.; Padar, A.; Huang, C.X.; Cunnigham, H.T.; Farinas, A.J.; Milchgrub, S.; Euhus, D.M.; Gilcrease, M.; et al. Aberrant methylation of the adenomatous polyposis coli (APC) gene promoter 1A in breast and lung carcinomas. Clin. Cancer Res. 2001, 7, 1998-2004.

42. Trinh, B.N.; Long, T.I.; Laird, P.W. DNA methylation analysis by MethyLight technology. Methods 2001, 25, 456-462. [CrossRef]

43. Zhao, X.; Jen, J.; Peikert, T.; Edell, E.; Tian, S.; Yang, P.; Huang, Y.; Zou, H. Selection of sensitive methylation markers for the detection of non-small cell lung cancer. J. Mol. Biomark. Diagn. 2015, 6, 1. [CrossRef] 
44. Hwang, S.H.; Kim, K.U.; Kim, J.E.; Kim, H.H.; Lee, M.K.; Lee, C.H.; Lee, S.Y.; Oh, T.; An, S. Detection of HOXA9 gene methylation in tumor tissues and induced sputum samples from primary lung cancer patients. Clin. Chem. Lab. Med. 2011, 49, 699-704. [CrossRef] [PubMed]

45. Wang, Y.; Yu, Z.; Wang, T.; Zhang, J.; Hong, L.; Chen, L. Identification of epigenetic aberrant promoter methylation of RASSF1A in serum DNA and its clinicopathological significance in lung cancer. Lung Cancer 2007, 56, 289-294. [CrossRef] [PubMed]

46. Lebanony, D.; Benjamin, H.; Gilad, S.; Ezagouri, M.; Dov, A.; Ashkenazi, K.; Gefen, N.; Izraeli, S.; Rechavi, G.; Pass, H.; et al. Diagnostic assay based on hsa-miR-205 expression distinguishes squamous from nonsquamous non-small-cell lung carcinoma. J. Clin. Oncol. 2009, 27, 2030-2037. [CrossRef] [PubMed]

47. Patnaik, S.; Mallick, R.; Kannisto, E.; Sharma, R.; Bshara, W.; Yendamuri, S.; Dhillon, S.S. MiR-205 and MiR-375 microRNA assays to distinguish squamous cell carcinoma from adenocarcinoma in lung cancer biopsies. J. Thorac. Oncol. 2015, 10, 446-453. [CrossRef]

(C) 2019 by the authors. Licensee MDPI, Basel, Switzerland. This article is an open access article distributed under the terms and conditions of the Creative Commons Attribution (CC BY) license (http://creativecommons.org/licenses/by/4.0/). 